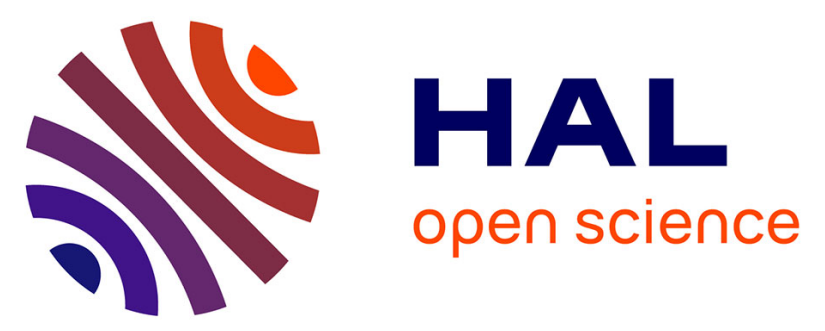

\title{
How Do Deep Convolutional SDM Trained on Satellite Images Unravel Vegetation Ecology?
}

Benjamin Deneu, Alexis Joly, Pierre Bonnet, Maximilien Servajean, François

Munoz

\section{- To cite this version:}

Benjamin Deneu, Alexis Joly, Pierre Bonnet, Maximilien Servajean, François Munoz. How Do Deep Convolutional SDM Trained on Satellite Images Unravel Vegetation Ecology?. ICPR 2020 - 25th International Conference on Pattern Recognition, Alberto Del Bimbo; Rita Cucchiara; Stan Sclaroff; Giovanni Maria Farinella; Tao Mei; Marco Bertini; Hugo Jair Escalante; Roberto Vezzani, Jan 2021, Milan / Virtual, Italy. pp.148-158, 10.1007/978-3-030-68780-9_15 . hal-03167637

\section{HAL Id: hal-03167637 \\ https://hal.inrae.fr/hal-03167637}

Submitted on 12 Mar 2021

HAL is a multi-disciplinary open access archive for the deposit and dissemination of scientific research documents, whether they are published or not. The documents may come from teaching and research institutions in France or abroad, or from public or private research centers.
L'archive ouverte pluridisciplinaire HAL, est destinée au dépôt et à la diffusion de documents scientifiques de niveau recherche, publiés ou non, émanant des établissements d'enseignement et de recherche français ou étrangers, des laboratoires publics ou privés. 


\title{
How do Deep Convolutional SDM trained on satellite images unravel vegetation ecology?
}

\author{
Benjamin Deneu ${ }^{1,2[0000-0003-0640-5706]}$, Alexis Joly ${ }^{1[0000-0002-2161-9940]}$, Pierre \\ Bonnet $^{2,3[0000-0002-2828-4389]}$, Maximilien Servajean ${ }^{[0000-0002-9426-2583]}$, and \\ François Munoz ${ }^{5[0000-0001-8776-4705]}$ \\ 1 INRIA, UMR LIRMM, Univ Montpellier, France \\ 2 AMAP, Univ Montpellier, CIRAD, CNRS, INRAE, IRD, Montpellier, France \\ 3 CIRAD, UMR AMAP, F-34398 Montpellier, France \\ 4 LIRMM, Université Paul Valéry, University of Montpellier, CNRS, Montpellier, France \\ 5 CNRS, LECA, France
}

\begin{abstract}
Species distribution models (SDM) assess and predict how species spatial distributions depend on the environment, due to species ecological preferences. These models are used in many different scenarios such as conservation plans or monitoring of invasive species. The choice of a model and of environmental data have strong impact on the model's ability to capture important ecological information. Specifically, state-of-the-art models generally rely on local, punctual environmental information, and do not take into account environmental variation in surrounding landscape. Here we use a convolutional neural network model to analyze and predict species distributions depending on high resolution data including remote sensing images, land cover and altitude. We show that the model unravel the functional response of vegetation to both local and large-scale environmental variation. To demonstrate the ecological significance of the results, we propose an original statistical analysis of t-SNE nonlinear dimension reduction. We illustrate and test the traitsspecies-environment relationships learned by the model and expressed in tSNE dimensions.
\end{abstract}

Keywords: Species Distribution Model · Convolutional Neural Network · Ecological Interpretation · Plant functional traits · Trait-environment relationships $\cdot \mathrm{t}-\mathrm{SNE}$

\section{Introduction}

Understanding and predicting the geographic distribution of species is a key objective in ecology and conservation. Species Distribution Models (SDM) aim at characterizing the relationship between the environment and species occurrences, depending on species ecological niches [14]. The ecological niche is multidimensional, and involves factors playing in a complex fashion (i.e. non linear system) and at multiple spatial scales. Most SDMs are correlative methods relating known species occurrence data to potential environmental predictors [17|1|25].Popular examples of such methods include MAXENT [26|27], random forest [9] and boosted regression trees [10]15]. Earlier works devised SDMs based on single-layer neural networks 
[22|2], and recently deep neural networks have proved relevant to better address the complexity of ecological niches, allowing to recognized a larger complexity in the way environment shapes ecological niches [6|4]. Key advantages of deep learning are that (i) it allows characterizing complex structuring of ecological niche depending on multiple environmental factors, (ii) it can learn ecological features common to a large number of species, and thus grasp the signatures of common ecological processes and improve SDM predictions across species [5]7].

A specific class of neural networks initially proposed in [21], named Convolutional Neural Networks (CNN), has very recently been proposed for SDM [5]13]. A property of $\mathrm{CNN}$ is that they rely on spatial environmental tensors rather than on local values of environmental factors. These tensors represent the spatial realization of environmental factors around each point. Unlike other SDM approaches, CNNbased SDMs (CNN-SDMs) can use this very large input data and therefore potentially capture richer information than in punctual vectors. CNNs were originally designed for image classification [21] and proved to outperform any other statistical or machine learning methods in the task of learning complex visual patterns. This largely explains why $\mathrm{CNN}$-based architectures are the most popular deep learning implementations in ecological studies since few years [7].

In this paper, we use a deep species distribution model based on a convolutional neural network applied to high resolution remote sensing images as one of the input covariables. This model has already been evaluated and compared to other more classical methods revealing superior performance to state of the art models. In this paper we are interested in the interpretation of these performances. The major contribution of the paper is to provide an ecological interpretation of the model. Therefore, we propose to rely on t-SNE [23], a nonlinear dimension reduction technique widely used in data science to visualize high-dimensional feature spaces. More precisely, we use it to build a low-dimensional embedding of the 2028-dimensional representation space learned by the convolutional neural network (i.e. the 2028 neurons used as input of the species prediction layer of the model). The resulting lowdimensional embedding is then used in two ways: (i) to visualize the relationships between the learned features, the species traits and the environment, and (ii) to quantify these relationships using statistical tests. The main outcome of our study is that the statistical tests clearly demonstrate that the model was able to capture meaningful relationships between the species traits and the environment. This is particularly remarkable in the sense that none of these information were used as input covariables during the training of the model. The model was actually capable of inferring them directly from the high-resolution spatialized images used as input and the species occurrences.

\section{Material and Methods}

\subsection{CNN-SDM Model Training and Validation}

Training Dataset A detailed description of the dataset (named GeoLifeCLEF 2020) is provided in [8]. It consists of 1,921,123 observations from the US $(1,097,640)$ and France $(823,483)$ covering 31,435 plant and animal species (Table 1 . Each species 
observation is paired with high-resolution covariates (RGB-IR imagery, land cover and altitude), see Fig 1 . We used RGB-IR imagery from the 2009-2011 cycle of the National Agriculture Imagery Program (NAIP) in the U.S ${ }^{6}$ and from the BD-ORTHO ${ }^{\circledR}$ 2.0 and ORTHO-HR® 1.0 databases from IGN in France 7 Land cover data originates from the National Land Cover Database (NLCD) [20] for the U.S. and from CESBIO ${ }^{8}$ for France. All elevation data comes from the NASA Shuttle Radar Topography Mission (SRTM) ${ }^{9}$ All of these high-resolution covariates were homogenized at a spatial resolution of 1 meter per pixel and provided as $256 \times 256$ images covering a $256 \mathrm{~m}$ $\times 256 \mathrm{~m}$ square centered on each observation (some were oversampled and some where downsampled).

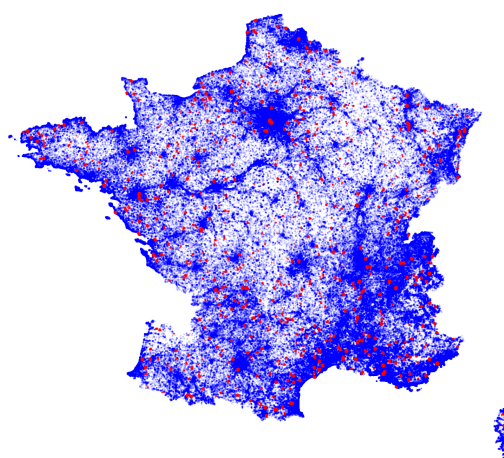

(a) Occurrences

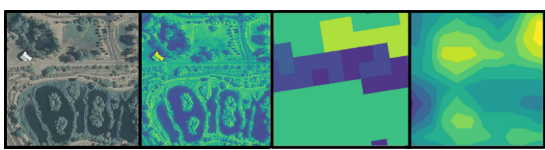

(b) Example of high-resolution covariates patch (respectively: RGB imagery, IR imagery, altitude, land cover).

Fig. 1: Occurrences distribution (training data in blue, test data in red) in France (a), and an example high-resolution tensor (b). RGB patch is displayed with native colors, IR imagery, altitude and land cover are in artificial colors from purple (lowest values) to yellow (highest values).

Table 1: Number of occurrences, of species and of occurrences per species grouped for plants and animals, and per region (for the training set).

\begin{tabular}{l|cc|cc|cc}
\hline \multirow{2}{*}{ Kingdom } & \multicolumn{2}{|c|}{ Nb. of occurrences } & \multicolumn{2}{c|}{ Nb. of species } & \multicolumn{2}{c}{ Nb. of occ. per sp. } \\
\cline { 2 - 7 } & US & France & US & France & US & France \\
\hline Plants & 524,280 & 741,010 & 11,369 & 3,114 & 46.1 & 238.0 \\
Animals & 551,563 & 61,865 & 13,882 & 4,899 & 39.7 & 12.6 \\
All & $1,622,120$ & 802,875 & 25,251 & 8,013 & 42.6 & 100.2 \\
\hline
\end{tabular}

6 National Agriculture Image Program, https://www.fsa.usda.gov

7 https://geoservices.ign.fr

8 http://osr-cesbio.ups-tlse.fr/ oso/posts/2017-03-30-carte-s2-2016/

9 https://lpdaac.usgs.gov/products/srtmgl1v003/ 
Deep Convolutional SDM Architecture Our deep convolutional neural network is the composition of non linear transformations of the input space $z=\phi(x)$ with a linear classifier $\psi(z)$. The vector $z$ is called the representation vector, or feature vector of the input tensor $\boldsymbol{x}$. The architecture used is based on the Inception V3 [28] model adapted in the same way introduced in [11]. The representation layer has size 2048 and the loss is the cross-entropy. The output of the model can be interpreted as the probability for each species.

Predictive Performance We evaluated the model and compared it to other more classical approaches, such as a random forest learned on environmental data (RF), using a spatial block holdout procedure ${ }^{10}$ On a top-30 score, the CNN obtained $23.5 \%$ success compared to the $20.4 \%$ success of the random forest environmental model. This evaluation allowed us to validate the performance of the model (CNNSDM) that we will study later in this report.

\subsection{Ecological Interpretation of the Learned Features}

We considered environmental and species trait data not used during model training. We assess the extent to which the model could capture ecological information such as the functional response of plant species to environmental constraints (specifically, climatic and pedological factors). In the following subsections, we first describe the environmental and species traits data used 2.3. In subsection 2.4. we then describe the non linear dimension reduction technique that we used to embed the 2048-dimensional feature vectors $\mathbf{z}$ into a low-dimensional space of 2 or $3 \mathrm{di}$ mensions aimed at conserving only the most structuring information. In subsection 2.5. we describe the statistical tests that were performed on top of the resulting lowdimensional embedding for the ecological interpretation. Finally, in subsection 2.6. we describe some additional methodological details that were used for visualization purposes.

\subsection{Environmental and Trait Data}

We used 19 bio-climatic rasters (30 $\operatorname{arcsec}^{2} /$ pixel (above $1 \mathrm{~km}^{2} /$ pixel) from WorldClim [19]) and 8 pedologic rasters $\left(250 \mathrm{~m}^{2} /\right.$ pixel, from SoilGrids [18), the detailed list and resolutions are presented in Table 2 .

We used ecological information available for more than 1,400 plant species, in terms of Ellenberg's indicator values (EIVs) [16] (see Table3. These variables represent an ordinal classification of ecological strategies for major environmental constraints and the use of essential resources [3].

$\overline{10}$ Test occurrences are contained in $5 \times 5 \mathrm{~km}$ quadrats with no train occurrences and represent $2.5 \%$ of the overall set. 
Table 2: Summary of environmental variable rasters provided.

\begin{tabular}{|r|l|c|}
\hline Name & Description & Res. \\
\hline bio_1 & Annual Mean Temperature & 30 arcsec \\
bio_2 & Mean Diurnal Range (Mean of monthly (max temp - min temp)) & 30 arcsec \\
bio_3 & Isothermality (bio_2/bio_7) (* 100) & 30 arcsec \\
bio_4 & Temperature Seasonality (standard deviation *100) & 30 arcsec \\
bio_5 & Max Temperature of Warmest Month & 30 arcsec \\
bio_6 & Min Temperature of Coldest Month & 30 arcsec \\
bio_7 & Temperature Annual Range (bio_5-bio_6) & 30 arcsec \\
bio_8 & Mean Temperature of Wettest Quarter & 30 arcsec \\
bio_9 & Mean Temperature of Driest Quarter & 30 arcsec \\
bio_10 & Mean Temperature of Warmest Quarter & 30 arcsec \\
bio_11 & Mean Temperature of Coldest Quarter & 30 arcsec \\
bio_12 & Annual Precipitation & 30 arcsec \\
bio_13 & Precipitation of Wettest Month & 30 arcsec \\
bio_14 & Precipitation of Driest Month & 30 arcsec \\
bio_15 & Precipitation Seasonality (Coefficient of Variation) & 30 arcsec \\
bio_16 & Precipitation of Wettest Quarter & 30 arcsec \\
bio_17 & Precipitation of Driest Quarter & 30 arcsec \\
bio_18 & Precipitation of Warmest Quarter & 30 arcsec \\
bio_19 & Precipitation of Coldest Quarter & 30 arcsec \\
orcdrc & Soil organic carbon content (g/kg at $15 \mathrm{~cm} \mathrm{depth)}$ & $250 \mathrm{~m}$ \\
phihox & Ph x 10 in H20 (at 15cm depth) & $250 \mathrm{~m}$ \\
cecsol & Cation exchange capacity of soil in cmolc/kg 15cm depth & $250 \mathrm{~m}$ \\
bdticm & Absolute depth to bedrock in cm & $250 \mathrm{~m}$ \\
clyppt & Clay (0-2 micro meter) mass fraction at 15cm depth & $250 \mathrm{~m}$ \\
sltppt & Silt mass fraction at 15cm depth & $250 \mathrm{~m}$ \\
sndppt & Sand mass fraction at 15cm depth & $250 \mathrm{~m}$ \\
bldfie & Bulk density in kg/m3 at 15cm depth & $250 \mathrm{~m}$ \\
\hline
\end{tabular}

Table 3: Summary of the used plant species traits.

\begin{tabular}{|l|l|l|}
\hline Name & Description & Ranges of values (Nb species) \\
\hline EIV L & Light availability & $2-9(1,423)$ \\
EIV T & Temperature & $1-9(1,413)$ \\
EIV K & Climatic continentality & $1-8(1,411))$ \\
EIV AirH & Air humidity & $1-9(1,405)$ \\
EIV F & Soil humidity & $1-12(1,405)$ \\
EIV R & Reaction (soil acidity / pH) & $1-9(1,410)$ \\
EIV TroL & Trophic level & $1-9(1,412)$ \\
EIV S & Salt (soil salinity) & $0-9(1,416)$ \\
EIV SoiT & Soil texture & $1-9(1,416)$ \\
EIV N & Nitrogen (soil fertility) & $1-9(1,422)$ \\
\hline
\end{tabular}




\subsection{Dimension Reduction}

CNNs train a representation space in which species occurrences projections $\mathbf{z}=\phi(\mathbf{x})$ tend to be linearly separable (thanks to the multinomial logistic regression $\psi$ trained on top of $\mathbf{z}$ ). We analyzed how the structure of the learned space could grasp major ecological and environmental information unused during learning. We identified major dimensions of the learned space by projecting the feature vectors $\mathbf{z}$ into a very low-dimensional space of 2 or 3 dimensions. For this purpose, we used the $\mathrm{t}$-SNE dimension reduction method 23 . First, t-SNE constructs a probability distribution over pairs of high-dimensional objects such as similar objects are assigned a higher probability while dissimilar points are assigned a lower probability. Second, tSNE defines a similar probability distribution over the points in the low-dimensional map, and it minimizes the Kullback-Leibler divergence (KL divergence) between the two distributions with respect to the locations of the points in the map. The main advantage of $\mathrm{t}-\mathrm{SNE}$ is that it is able to preserve the main regularities of the original space even if some of them are not linear. We also computed PCA as a preliminary dimension reduction step.

In more details, to process the dimension reduction we randomly selected 32,000 training occurrences $\mathbf{x}^{j}$ and computed their representations $\mathbf{z}^{j}=\phi\left(\mathbf{x}^{j}\right)$. Then, we first reduced the dimension from 2048 to 50 by PCA using the PCA method of the scikitlearn package. The resulting 50-dimensional feature vectors were then further reduced by t-SNE using the t-SNE method of the scikitlean package. For most experiments, the used dimension for t-SNE was set to 2 (apart from the geographical map of Figure Figure $3 \mathrm{~b}$ where it was set to 3 and base on other occurrences, see section subsection 2.6. In the following, we denote as $\tilde{\mathbf{z}}=g(\mathbf{z})$ the resulting 2dimensional feature vectors where the function $g$ denotes the complete dimension reduction function (PCA+t-SNE).

\subsection{Ecological Interpretation of t-SNE Dimensions}

Relationship of t-SNE Dimensions to Ecological Traits and Environmental Factors For each of the two variables $\tilde{\mathbf{z}}_{1}$ and $\tilde{\mathbf{z}}_{2}$, corresponding in the two axis of the t-SNE, we fitted a linear model using either the environmental variables or the species trait variables as input covariates ( $\mathrm{lm}$ function in R environment).

The link between the representation space learned by the model (condensed in the t-SNE space) and these data (not used during the learning process) offers a way to interpret the ecological significance of patterns learned by the CNN-SDM models.

Traits-Environment Relationships Trait-environment relationship represent essential functional responses of plants to changing environmental conditions. Characterizing these relationships is an essential goal in functional biogeography [29]. We tested whether t-SNE axes unravel such relationships by mean of weighted correlation analysis. We calculated the correlation between species trait and environmental value for each training occurrence, weighted by the score of the occurrence along each t-SNE axis. Under the null hypothesis, the t-SNE axis does not reflect any linkage between trait and environment, under the alternative hypothesis, increasing score 
is associated to joint variation of trait and environment. We measured the weighted correlation [24] for each t-SNE variable $\tilde{\mathbf{z}}_{k}$ and for each pair of ecological trait and environmental variable. We used the function wtd.cor package weights in R. Furthermore, because we computed many tests for all pairs of trait and environmental variables, we corrected the p-value of the tests by the method false discovery rate (p.adjust function in R).

\subsection{Visualization}

We used different types of visualisations to illustrate the main sources of ecological variation expressed in the two-dimensional t-SNE space. We discretized the space of the t-SNE by applying an $n \times n$-sized grid mesh. For each cell of this grid we retrieved the $\mathrm{m}$-coordinate vector of the center of the cell and we search for the nearest neighbor among the set of vectors $\tilde{\mathbf{z}}$ using scikitlearn's nearest neigbhor function. Once the closest point to the center of each cell is retrieved, only those that are actually inside the respective cell are kept by a filter on these coordinates. This results in associating for each cell the occurrence closest to the center if there is one. The different figures of this visualization consist in the display, for each cell, of a data relative to the associated occurrence. For the first fig. 3a we displayed the RGB patch of the remote sensing imagery corresponding to this occurrence. For the other figures 2a and $2 \mathrm{~b}$ we displayed the value of an ecological trait of the corresponding species or the realization of one of the environmental variables at the point of occurrence.

The second representation is based on the 3-dimensional t-SNE space and, conversely, represents the realization of this space on the geographical space. This 3dimensional t-SNE space was obtained by the same process as the 2-dimensional space described in detail in section 2.4 but with one difference, the dimension reduction was not performed with the same set of occurrences. As this representation is geographical, it requires a better coverage of the territory than the simple random selection of a certain number of occurrences. We selected occurrences with a method of nearest neighbor. For a grid of points with a resolution of $1 \mathrm{~km}$ over the whole territory, we associated each point to the nearest occurrence. The selected occurrences are those used for dimension reduction. Then to plot the 3-dimensional t-SNE on the map, we re-scale each axis such as the values are included in 0-255. Each point can then be associated to a RGB color by its coordinates. The resulting map is the plot of each point as a color pixel at its geographical position.

\section{Results and Discussion}

The statistical analysis of the learned t-SNE space reveals that the CNN-based SDM could grasp important ecological processes shaping the large scale distributions of plants. Indeed, almost all linear models linking scores along t-SNE to species traits provided highly significant relationships (Table 4 ). We can in particular note a strong coefficient on EIV T (species preferences in Temperature) on both axes. The plant Ellenberg indicator values could alone explain a large part of the variation of species scores along t-SNE axes (Adjusted $\mathrm{R}^{2}$ of 0.111 and 0.231). These results show that 
Table 4: Ellenberg linear models

\begin{tabular}{lcc}
\hline & tsne_1 & tsne_2 \\
& Estimate (Std. Error) & Estimate (Std. Error) \\
\hline EIV L & $-1.562^{* * *}(0.236)$ & $2.630^{* * *}(0.214)$ \\
EIV T & $-5.035^{* * *}(0.260)$ & $9.478^{* * *}(0.235)$ \\
EIV K & $2.523^{* * *}(0.389)$ & $0.150(0.352)$ \\
EIV AirH & $0.732^{*}(0.378)$ & $-0.869^{* *}(0.341)$ \\
EIV F & $1.461^{* * *}(0.409)$ & $2.328^{* * *}(0.370)$ \\
EIV R & $0.461^{* *}(0.213)$ & $-0.686^{* * *}(0.192)$ \\
EIV TroL & $2.902^{* * *}(0.181)$ & $3.851^{* * *}(0.163)$ \\
EIV S & $-2.714^{* * *}(0.366)$ & $-0.981^{* * *}(0.331)$ \\
EIV SoiT & $1.649^{* * *}(0.252)$ & $2.848^{* * *}(0.228)$ \\
EIV N & $0.211(0.212)$ & $-0.598^{* * *}(0.192)$ \\
Constant & $-5.951(3.947)$ & $-113.831^{* * *}(3.569)$ \\
\hline $\mathrm{R}^{2}$ & 0.111 & 0.232 \\
Adjusted $\mathrm{R}^{2}$ & 0.111 & 0.231 \\
\hline Note: & & ${ }^{*} \mathrm{p}<0.1 ;{ }^{* *} \mathrm{p}<0.05 ;{ }^{* * *} \mathrm{p}<0.01$
\end{tabular}

the model is able to capture information related to the ecology of the species in the patches.

Fig. 2a represent the species preferences in temperature and Fig. 2b represent Annual mean temperature of occurrences data on the t-SNE. We found a clear gradient of mean annual temperature on the t-SNE. The fact that this gradient is particularly sharp on t-SNE, which is the result of a dimension reduction, indicates that information strongly correlated with annual temperature is captured and important in the model. It is quite logical that the figure on the associated ecological trait (EIV $\mathrm{T}$ ) is similar confirming that the plants with the coolest temperature preferences are found in the coldest areas and vice versa. These results highlight that the information captured by the model is strongly related to the environment and ecology of the species, even though this data was not used directly. Fig. 3a represent RGB patches from the model training data on the t-SNE space and Fig. $3 \mathrm{~b}$ represents the geographical projection of the 3-dimensional t-SNE space on the territory with the colors defined by the position in the t-SNE space.

These two figures highlight two complementary pieces of information. On the one hand, the display of the patches on the t-SNE highlights the landscape factors that the CNN is able to identify and differentiate in its representation space. Indeed the Fig. 3a highlights different areas of the t-SNE corresponding to several major types of distinct landscapes. We can note for example the mountain areas on the left next to which we find the forests a little lower down. The more agricultural areas also stand out while the lower and right part of the t-SNE is dominated by urban type landscapes. On the other hand, Fig. $3 \mathrm{~b}$ shows learning on a larger scale. This map shows how the $\mathrm{CNN}$ has learned to distinguish large eco-geographic regions. Of particular note is the Mediterranean region (on southern part of the map), and the 


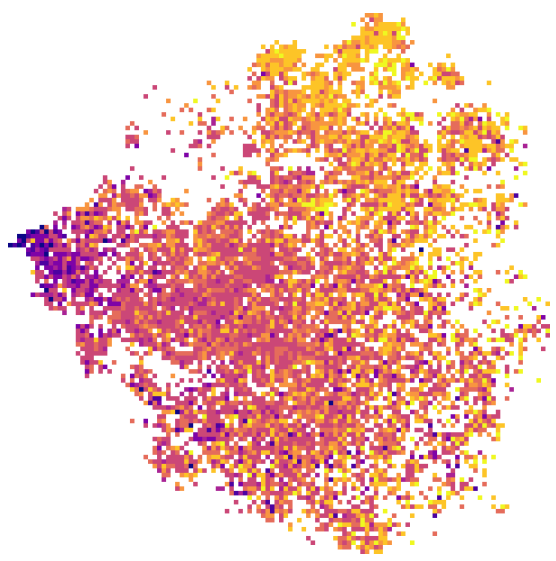

(a)

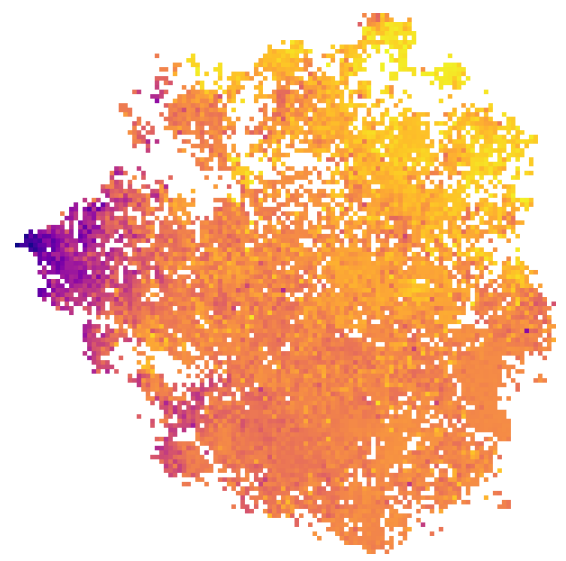

(b)

Fig. 2: Visualization of the occurrences' species preferences in temperature (EIVT)(a) and the annual mean temperature (bio_1) at occurrences location (b) on the t-SNE space. Artificial colors from purple (lowest values) to yellow (highest values).

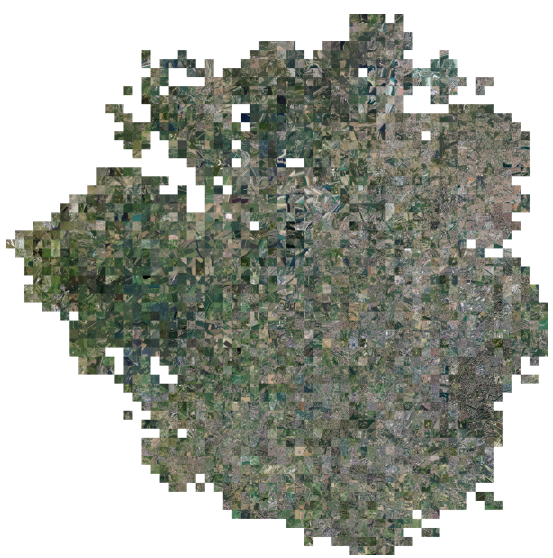

(a)

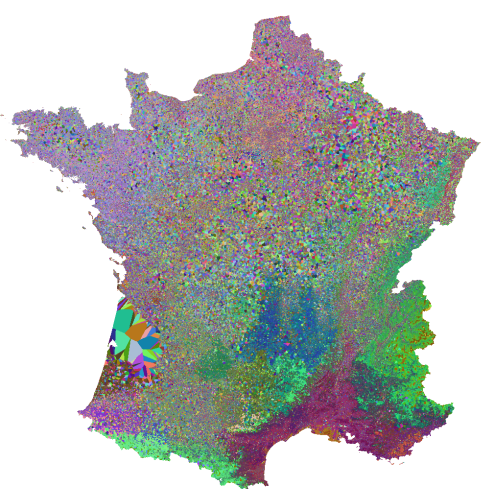

(b)

Fig. 3: (a) Visualization of the remote sensing imagery patch (RGB) of occurrences on the t-SNE space. (b) Geographical projection of the 3-dimensional t-SNE space on the territory.

mountainous areas (i.e. the Pyrenees on the south-western part, and the Alps on the south-eastern part), which seem to be well identified. More generally the map allows to show coherence on a large scale, between typical vegetation zones well identified by ecologists, and bio-geographic zones well characterized on the field in previous studies. The confrontation of these two results shows that the model is capable of discerning both large regions and different habitats within the same large geograph- 
ical area (such as urban-rural). The ability to identify factors at different scales based on fine scale imaging training data is an important result that highlights one of the advantage of the CNN models. Concerning the species trait-environment relationships, the detailed results matrix of each pair for each axis is given in supplementary materials Tables $\mathrm{C} 1$ and $\mathrm{C} 2[12]$. A general finding is that most of the species traitenvironment correlations weighted by the t-SNE axes are highly significant. This shows that the $\mathrm{CNN}$ is capable of learning a relationship between environment and species ecology through the use of high-resolution covariate patches. All these results highlight a strong point of the $\mathrm{CNN}$ model which is capable of capturing information related to the environment and the ecology of species through the use of data accessible at large scale and fine resolution, but also to highlight links between the environment and the ecology of species. This model could therefore be useful in many ecological scenarios where the objective would be to study this link. All these points are also insights for interpreting the performance of the CNN models. By learning a representation space common to all the studied species, the model is able to extract common information between species that are directly correlated to environmental factors or related to the ecology of the species.

\section{Conclusion}

Our study shows the interest of using convolutional neural networks for species distribution modelling (CNN-SDM), at high spatial resolution over large geographical areas. The use of the t-SNE technique made it possible for the first time to visualise and test the learning capacities of this type of model to capture relationships between ecological strategies of plants and environmental conditions at their occurrence locations. The methodological framework established here offers a new way of statistically assessing the extent to which the relationships between the plant traits and their environments are correlated, inspired by the conceptual and methodological framework of functional biogeography [29]. It also allows visualizing through tSNE the clusters of visual information (from satellite images) deemed most relevant during the learning phase of the CNN-SDMs. This work shows that the approach captures richer information on the landscape context than the specific ponctual data related to the environment for predicting the presence of species, and will undoubtedly open up new perspectives in the analysis of plant-environment trait relationships.

\section{Acknowledgement}

This project has received funding from the French National Research Agency under the Investments for the Future Program, referred as ANR-16-CONV-0004 and from the European Union's Horizon 2020 research and innovation program under grant agreement No 863463 (Cos4Cloud project). 


\section{References}

1. Antoine, G., Wilfried, T.: Predicting species distribution: offering more than simple habitat models. Ecology Letters 8(9), 993-1009 (2005). https://doi.org/10.1111/j.14610248.2005.00792.x

2. Baran, P., Lek, S., Delacoste, M., Belaud, A.: Stochastic models that predict trout population density or biomass on a mesohabitat scale. Hydrobiologia 337(1), 1-9 (Nov 1996). https://doi.org/10.1007/BF00028502

3. Bartelheimer, M., Poschlod, P.: Functional characterizations of ellenberg indicator valuesa review on ecophysiological determinants. Functional ecology 30(4), 506-516 (2016)

4. Benkendorf, D.J., Hawkins, C.P.: Effects of sample size and network depth on a deep learning approach to species distribution modeling. Ecological Informatics 60, 101137 (2020)

5. Botella, C., Joly, A., Bonnet, P., Monestiez, P., Munoz, F.: A deep learning approach to species distribution modelling. Multimedia Technologies for Environmental \& Biodiversity Informatics (2018)

6. Chen, D., Xue, Y., Chen, S., Fink, D., Gomes, C.P.: Deep multi-species embedding. CoRR abs/1609.09353 (2016), http://arxiv.org/abs/1609.09353

7. Christin, S., Hervet, É., Lecomte, N.: Applications for deep learning in ecology. Methods in Ecology and Evolution 10(10), 1632-1644 (2019)

8. Cole, E., Deneu, B., Lorieul, T., Servajean, M., Botella, C., Morris, D., Jojic, N., Bonnet, P., Joly, A.: The geolifeclef 2020 dataset. arXiv preprint arXiv:2004.04192 (2020)

9. Cutler, D.R., Edwards Jr., T.C., Beard, K.H., Cutler, A., Hess, K.T., Gibson, J., Lawler, J.J.: Random forests for classification in ecology. Ecology 88(11), 2783-2792 (2007). https://doi.org/10.1890/07-0539.1

10. De'ath, G.: Boosted trees for ecological modeling and prediction. Ecology 88(1), 243-251 (2007).https://doi.org/10.1890/0012-9658(2007)88[243:BTFEMA]2.0.CO;2

11. Deneu, B., Servajean, M., Joly, A.: Participation of lirmm/inria to the geo-lifeclef 2020 challenge. CLEF working notes (2020)

12. Deneu, B., Joly, A., Bonnet, P., Servajean, M., Munoz, F.: Supplementary materials: How do deep convolutional sdm trained on satellite images unravel vegetation ecology?, https : //gitlab.inria.fr/bdeneu/supplementary-materials-maes2020-paper-19

13. Deneu, B., Servajean, M., Botella, C., Joly, A.: Location-based species recommendation using co-occurrences and environment- geolifeclef 2018 challenge. In: CLEF working notes 2018 (2018)

14. Elith, J., Leathwick, J.R.: Species Distribution Models: Ecological Explanation and Prediction Across Space and Time. Annual Review of Ecology, Evolution, and Systematics 40, 677-697 (2009)

15. Elith, J., Leathwick, J.R., Hastie, T.: A working guide to boosted regression trees. Journal of Animal Ecology 77(4), 802-813 (2008). https://doi.org/10.1111/j.1365-2656.2008.01390.x

16. Ellenberg, H.H.: Vegetation ecology of central Europe. Cambridge University Press (1988)

17. Guisan, A., Zimmermann, N.E.: Predictive habitat distribution models in ecology. Ecological Modelling 135(2), 147 - 186 (2000). https://doi.org/https://doi.org/10.1016/S03043800(00)00354-9

18. Hengl, T., de Jesus, J.M., Heuvelink, G.B., Gonzalez, M.R., Kilibarda, M., Blagotić, A., Shangguan, W., Wright, M.N., Geng, X., Bauer-Marschallinger, B., et al.: Soilgrids250m: Global gridded soil information based on machine learning. PLoS one 12(2) (2017)

19. Hijmans, R.J., Cameron, S.E., Parra, J.L., Jones, P.G., Jarvis, A.: Very high resolution interpolated climate surfaces for global land areas. International Journal of Climatology: A Journal of the Royal Meteorological Society 25(15), 1965-1978 (2005) 
20. Homer, C., Dewitz, J., Yang, L., Jin, S., Danielson, P., Xian, G., Coulston, J., Herold, N., Wickham, J., Megown, K.: Completion of the 2011 national land cover database for the conterminous united states-representing a decade of land cover change information. Photogrammetric Engineering \& Remote Sensing 81 (5), 345-354 (2015)

21. LeCun, Y., Boser, B., Denker, J.S., Henderson, D., Howard, R.E., Hubbard, W., Jackel, L.D.: Backpropagation applied to handwritten zip code recognition. Neural computation 1(4), 541-551 (1989)

22. Lek, S., Delacoste, M., Baran, P., Dimopoulos, I., Lauga, J., Aulagnier, S.: Application of neural networks to modelling nonlinear relationships in ecology. Ecological Modelling 90(1), 39 - 52 (1996). https://doi.org//10.1016/0304-3800(95)00142-5

23. Maaten, L.v.d., Hinton, G.: Visualizing data using t-sne. Journal of machine learning research 9(Nov), 2579-2605 (2008)

24. Miller, J.E., Damschen, E.I., Ives, A.R.: Functional traits and community composition: a comparison among community-weighted means, weighted correlations, and multilevel models. Methods in Ecology and Evolution 10(3), 415-425 (2019)

25. Peterson, A.T.: Ecological niches and geographic distributions. Princeton University Press (2011)

26. Phillips, S.J., Anderson, R.P., Schapire, R.E.: Maximum entropy modeling of species geographic distributions. Ecological modelling 190(3-4), 231-259 (2006)

27. Phillips, S.J., Dudík, M.: Modeling of species distributions with maxent: new extensions and a comprehensive evaluation. Ecography 31(2), 161-175 (2008). https://doi.org/10.1111/j.0906-7590.2008.5203.x

28. Szegedy, C., Vanhoucke, V., Ioffe, S., Shlens, J., Wojna, Z.: Rethinking the inception architecture for computer vision. In: Proceedings of the IEEE conference on computer vision and pattern recognition. pp. 2818-2826 (2016)

29. Violle, C., Reich, P.B., Pacala, S.W., Enquist, B.J., Kattge, J.: The emergence and promise of functional biogeography. Proceedings of the National Academy of Sciences 111(38), 13690-13696 (2014) 